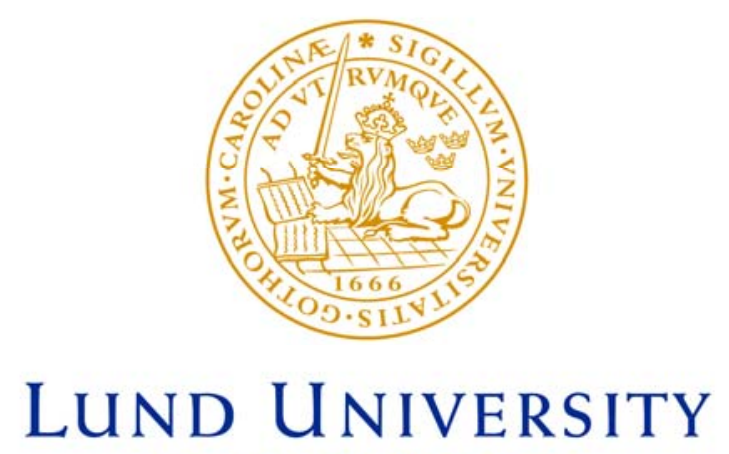

Faculty of Medicine

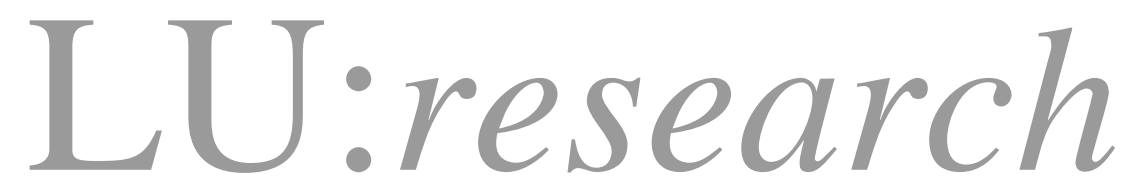

Institutional Repository of Lund University

This is an author produced version of a paper published in Journal of clinical gastroenterology. This paper has been peer-reviewed but does not include the final publisher proof-corrections or journal pagination.

Citation for the published paper:

Ohlsson, Bodil.

"Bowel habits in women during pregnancy, breast

feeding, and after breast feeding "

Journal of clinical gastroenterology, 2007, Vol: 41, Issue: 2, pp. 224-5.

http://dx.doi.org/10.1097/01.mcg.0000225512.82794.29

Access to the published version may require journal subscription.

Published with permission from: Wolters Kluwer 


\section{Bowel habits in women during pregnancy, breast feeding and after breast feeding}

\section{To the Editor;}

The etiology of functional disorders continues to be debated. Since more women than men suffer from IBS, the role of sex hormones has been raised. Clinically, we have heard some women reporting improvement of their constipation during breast feeding. The aims of the present study were therefore to; (1) prospectively follow healthy women through pregnancy, breast feeding and after breast feeding and examine whether the bowel habits and abdominal pain varies during this period, and to; (2) retrospectively examine the bowel habits of patients with chronic constipation in these circumstances.

Healthy women attending to the maternity clinic in the early phase of pregnancy were asked to participate. Twenty-one women, mean age $31 \pm 5$ years, were willing to complete the questionnaire about their bowel habits for one week on 3 different occasions, namely, during their pregnancy (week 30-36); while breast feeding (2 months after delivery); and at two months after breast feeding had been discontinued. Stool frequency was registered daily, as well as stool consistency, ranked according to the Bristol stool form scale (1). The sensation of incomplete evacuation and anorectal obstruction, and a need for manual maneuvers to facilitate defecation were answered by yes or no. The number of yes/week was used for the statistical calculations. Their subjective experience of abdominal pain, constipation and gas during the last month was ranked for the same week on a scale between $0-4$, where 0 was no report of trouble and 4 was very severe trouble. Values are given as the mean \pm standard deviation (SD). Friedman's test was used for comparisons between the different conditions.

Three of the women fulfilled the symptom criteria for Irritable Bowel Syndrome (IBS). There was a trend towards more abdominal pain during pregnancy than during and after breast feeding $(\mathrm{p}=0.055)$, and the symptom of gas was more troublesome during 
pregnancy than during the other periods $(\mathrm{p}=0.017)$. The women did not experience any difference in constipation during pregnancy and breast feeding compared to afterwards, although there was a firmer stool consistency during breast feeding $(\mathrm{p}=0.040)$ and a sensation of anorectal obstruction during pregnancy $(\mathrm{p}=0.028)$. Fifty-three women with chronic constipation (22 with slow transit constipation (STC)), mean age $47 \pm 12$ years, reported how their overall bowel habits had been during the major part of their pregnancy and breast feeding period, and during their menstrual cycle, before being included in a drug-trial (2). Of these, 38 women had experience of both pregnancy and breast feeding. Two patients improved while for two their constipation became aggravated during pregnancy, whereas three patients improved and one grew worse during breast feeding. Twelve women (23\%) experienced an improvement of their constipation during the menstrual phases (7 with STC), whereas 2 (5\%) were even more constipated during this phase. Two of the women (5\%) had more trouble with their constipation during the luteal phases. There was no correlation between a change of bowel habits during the menstrual cycle and during pregnancy and breast feeding (data not shown).

The results showed increased symptoms of gas and a sensation of anorectal obstruction during pregnancy, whereas breast feeding led to hardening of the stool in healthy women, albeit these conditions had no obvious effects in constipated women. Although it is a well established fact that pregnancy leads to constipation, only a few studies have examined this. In one retrospective study, the stool frequency was decreased in $11 \%$ of cases, whereas the stool frequency was increased in 34\% (3), and another study showed a prevalence of constipation in 31\% (4). These prevalences of constipation should be compared to those of the general population where the prevalence is almost $20 \%$ (5). The only prospective study found reported a prevalence of constipation in about $4.5 \%$ of women during both late pregnancy, breast feeding and at 12 months postpartum (NS), where the most striking finding was the 
frequent sensation of anorectal obstruction already early in the pregnancy (6). The sensation of anorectal obstruction in combination with an increased amount of gas found in the present study may be responsible for the assumption that constipation is a general problem during pregnancy.

Women suffering from IBS have a lower concentration in plasma of oxytocin than healthy controls (7). When we treated the constipated women with intranasal oxytocin, less abdominal pain and discomfort was seen among those who also suffered from IBS (2). In the present trial, which is the only one examining abdominal pain during pregnancy and breast feeding in healthy women, there was a trend towards more abdominal pain during late pregnancy, when the oxytocin levels are at the highest levels (8). Other hormonal changes, such as sexual hormones and insulin, occurring at the same time may counteract the effects of oxytocin (8). Further, the abdominal pain during pregnancy may have a different etiology than abdominal pain in IBS.

The positive effect of the menstrual phase on the constipation found in the present study is in agreement with earlier findings. Both healthy women, and women with IBS or pure constipation, reported that stool frequency is increased and stool consistency is looser during the menstrual phase, while stool consistency is harder during the luteal phase (9, 10, 11), explained as being due to high levels of progesterone and oestradiol during the luteal phase (9) and high prostaglandin production during the menstrual phase (12).

In conclusion, although it has been clinically observed that breast feeding improved constipation in some cases, this study failed to prove that this statement is of general validity and that pregnancy and breast feeding have positive effects on bowel habits and abdominal pain. On the other hand, the menstrual phase is often associated with a relief of constipation. 


\section{Bodil Ohlsson, M.D., Ph.D}

Department of Clinical Sciences

Malmö University Hospital

Entrance 35

S-205 02 Malmö, Sweden

Phone +46 40332323

fax +46 40336208

e-mail; bodil.ohlsson@med.lu.se

Acknowledgements: This study was supported by grants from the drug company Novo Nordic and the Development Foundation of Region Skåne. I would like to thank the staff at the maternity clinic in Hässleholm for recruiting subjects for this study.

The study protocols were approved by the local Ethics Committee at Lund University.

Written, informed consent was obtained from all subjects before their inclusion in the study. 


\section{References}

1. Lewis SJ, Heaton KW. Stool form scale as a useful guide to intestinal transit time. Scand J Gastroenterol 1997;32:920-924.

2. Ohlsson B, Truedsson M, Bengtsson M, et al. Effects of long-term treatment with oxytocin in chronic constipation; a double blind, placebo-controlled pilot trial. Neurogastroenterol Motil 2005;17:697-704.

3. Levy N, Lemberg E, Sharf M. Bowel habits in pregnancy. Digestion 1971;4:216-222.

4. Greenhalf JO, Leonard SD. Laxatives in the treatment of constipation in pregnant and breast-feeding mothers. The Practioner 1973;210:259-263.

5. Drossman DA, Li Z, Andruzzi E, et al. US householder survey of functional gastrointestinal disorders: prevalence, sociodemography and health impact. Dig Dis Sci 1993;38:1569-1580.

6. van Brummen HJ, Bruinse HW, van de Pol G, et al. Defecatory symptoms during and after the first pregnancy: prevalences and associated factors. Int Urogynecology $J$ [serial online]. March 2005;10.1007/s00192-005-1351-0. Available from: Springerlink

7. Uvnäs-Moberg K, Arn I, Theorell I, et al. Gastrin, somatostatin and oxytocin levels in the patients with functional disorders of the gastrointestinal tract and their response to feeding and interaction. J Psychosom Res 1991;35:525-533.

8. Silber M, Larsson B, Uvnäs-Moberg K. Oxytocin, somatostatin, insulin and gastrin concentrations vis-à-vis late pregnancy, breastfeeding and oral contraceptives. Acta Obstet Gynecol Scand 1991;70:283-289.

9. Davies GJ, Crowder M, Reid B, et al. Bowel function measurements of individuals with different eating patterns. Gut 1986;27:164-169. 
10. Houghton LA, Lea R, Jackson N, et al. The menstrual cycle affects rectal sensitivity in patients with irritable bowel syndrome but not healthy volunteers. Gut 2002;50:471-474.

11. Fukuda S, Matzuka M, Takahashi I, et al. Bowel habits before and during menses in Japanese women of climacteric age: A population based study. Tohoku J Exp Med 2005;206:99-104.

12. Arthur C, Ament ME, Song MK. Prostaglandin metabolism in relation to the bowel habits in women. Prostaglandins Leukot Essent Fatty Acids 1992;46:257-259. 